\title{
The Insects of Caja de Muertos Island, Puerto Rico'
}

\author{
Silverio Medina Gaud and Luis F. Martorell ${ }^{2}$
}

\section{INTRODUCTION}

Caja de Muertos is a small island located about 8 miles southeast of Ponce harbor (on the south coast of Puerto Rico) and approximately 5 miles from its shortest distance to the main south coast of the Island proper, that is, at latitude $17^{\circ} 54^{\prime}$ North and longitude $66^{\circ} 32^{\prime}$ West. It is 2 miles long and about one half mile at its widest, which is more or less at the center of the island, and has a total area of 404.65 cuerdas, $^{3}$ not counting the small area occupied by the lighthouse property.

\section{EARLY VISITS AND EXPLORATIONS}

Although, undoubtedly Caja de Muertos was used in the 16th and 17th centuries as a brief resting or hiding place for bucaneers, bandits, escaped convicts and fishermen, it became the site years later for scientific explorations and was visited by many scientists interested in the natural resources of the island.

One of the first to visit Caja de Muertos, probably for geographic and perhaps scientific purposes, was the famous French traveller-writer Labat (9), who spent 12 years in the Antillean Caribbean Islands during the period 1663 to 1705 . He carefully recorded the geography, took notes about the inhabitants, animals, crops and industries of all the places he visited; information very useful at the time.

Almost 2 centuries passed after Labat's visit to Caja de Muertos before it was explored March 9 to 11, 1915 by a group of botanists composed of N. L. Britton, J. F. Cowell, and Stewarson Brown (2). The plants recorded in this trip were included years later in Britton and Wilson's monumental work on the Botany of Puerto Rico and the Virgin Islands (3) and in Britton's own work on the same subject (1).

During June and July 1915, Marshall A. Howe studied the marine algae

1 Paper submitted to the Editorial Board August 16, 1973.

${ }^{2}$ Associate Entomologist and Professor Emeritus, respectively, Department of Entomology, Agricultural Experiment Station, Mayagüez Campus, University of Puerto Rico, Río Piedras, P.R. The authors are deeply indebted to the late Juan Alberto Wirshing of Ponce, P.R., for providing transportation and lodging during the different visits to that island by them and others accompanying the group. Without his encouragement and hearty cooperation this work would have not been possible.

${ }^{3} \mathrm{~A}$ cuerda is equivalent to 0.9712 acre. 
on the island, as well as the species found along the coast from Ponce to the southwestern end of Puerto Rico.

In 1962 two other American scientists, H. E. Anthony and G. G. Goodwin, visited Caja de Muertos in search of mammals and reptiles. Some of this material was sent to K. P. Schmidt, who was studying the herpetological fauna of Puerto Rico and the Virgin Islands (13) at the time.

On July 26, 1931 Chapman Grant and Cornelius Roosevelt collected reptiles there. Grant made another trip on August 20 of the same year (8).

\section{RECENT EXPLORATIONS}

Since 1931, Caja de Muertos has been visited by the following scientists:

Entomologists L. F. Martorell November 1947, and John S. Caldwell and L. F. Martorell, on December 5 and 11, 1947, to collect Cicadellidae and Fulgoridae.

Entomologists L. F. Martorell, S. Medina-Gaud, and E. A. JordánMusa, visited the island May 5, 1959. They visited the island again on May 24 to both collect and arrange for a larger group to make similar investigations later. This latter group visited the island May 27-30, 1959. S. Medina Gaud, L. F. Martorell, E. A. Jordán-Musa collected insects, A. Ayala Almodovar plant and free-living nematodes, and R. C. Woodbury and M. Cobin, plants.

L. F. Martorell, S. Medina Gaud and E. A. Jordán-Musa continued insect collecting on the island June 23-24, 1959, particularly at night by means of light traps. Martorell and Jordán-Musa spent a day collecting there December 9, 1960.

C. F. Smith, formerly Head, Department of Entomology, North Carolina State University, L. F. Martorell and M. E. Pérez-Escolar collected plant aphids there April 23, 1960. These aphid records were published by Smith et al. (14).

On July 21, 1966, a group composed of entomologists J. García-Tudurí and L. F. Martorell and botanists R. C. Woodbury and E. L. Little, Jr. visited the island to collect termites and plants.

The most recent trip to Caja de Muertos was made by $\mathrm{H}$. S. Telford, Entomologist at Washington State University, L. F. Martorell and J. García-Tuduri, to collect insects (arboreal termites and syrphid flies in particular) and plants. The syrphid records were published recently by Telford (15).

The 210 species recorded below are arranged in numerical order.

Unless otherwise noted, the following initials in parentheses designate the corresponding names of collectors: (LFM) for L. F. Martorell; (SMG) for S. Medina Gaud; (JGT) for J. C. García-Tuduri; and (EAJ) for E. A. Jordán-Musa. 


\section{ORDER ORTHOPTERA}

FAMILY BLATTIDAE

\section{Blatella germanica (L.)}

In kitchen, May 27-30, 1959, not very common, Acc. No. 270-594 (LFM, SMG).

2. Hemiblabera brunneri (Saussure)

One specimen collected, June 24, 1959, Acc. No. 274-59 (LFM, SMG, EAJ).

3. Periplaneta brunnea (Burmeister)

Three specimens, May 27-30, 1959, Acc. No. 269-59 (LFM, SMG).

4. Plecoptera dorsalis (Burmeister)

One specimen June 24, 1959, Acc. No. 272-59 (LFM, SMG); one specimen in the Station ${ }^{5}$ Collection, Nov. 1947, Acc. No. 270-47 (LFM).

FAMILY MANTIDAE

5. Callimantis antillarum (Saussure)

Fairly abundant on the branches and foliage of plants May 27-30, 1959 and June 24, 1959, Acc. No. 268-59 (LFM, SMG, EAJ); two specimens in the Station Collection, Nov. 1947, Acc. No. 270-47 (LFM).

\section{FAMILY TETTIGONIIDAE}

\section{Microcentrum triangulatum Brunner}

Among weeds, May 27-30, 1959, Acc. 257-59 (SMG, EAJ). Another specimen in Station Collection, Nov. 1947, Acc. 257-47 (LFM).

\section{FAMILY GRYLLIDAF}

7. Acheta asimilis $\mathrm{F}$.

Fairly abundant May 27-30, 1959 (LFM, SMG, EAJ).

8. Orocharis vaginalis Saussure

Collected May 27-30, 1959 and June 24, 1959, Acc. 256-59 and 257-59 (LFM, SMG, EAJ).

4 This and subsequent similar figures refer to the Accession Catalog number at the Agricultural Experiment Station's Insect Collection in Rio Piedras, indicating the specimen number and the year of collection.

"The word "Station" stands for Agricultural Experiment Station. 
9. Rhammatocerus cyanipes (F.)

Not abundant, May 5, 1959 and Dec. 9, 1960 (LFM, SMG, EAJ). One specimen, Station Collection, Nov. 1947, Acc. 263-47 (LFM).

10. Schistocerca columbina (Thunberg)

Collected May 27-30, 1959 and June 24, 1959, Acc. 258-59 (LFM, SMG, EAJ).

11. Schistocerca pallens (Thunberg)

One specimen May 27-30, 1959, Acc. 25-59 (LFM, SMG, EAJ).

12. Sphingonotus haitiensis (Saussure)

Two specimens collected by sweeping on grasses May 27-30, 1959 (LFM, SMG, EAJ).

\section{ORDER ISOPTERA}

\section{FAMILY TERMITIDAE}

\section{Nasutitermes nigriceps (Haldeman)}

The only arboreal termite present on Caja de Muertos Island, a species typical of xerophytic areas, recorded on the following host trees: Cassia macrantha, Bourreria succulenta, Bursera simaruba, Capparis flexuosa Coccoloba microstachya, Coccoloba uvifera, Comocladia dodonea, Conocarpus erecta, Elaeodendron xylocarpum, Exostema caribea, Ficus laevigata, Hippomane mancinella, Leucaena glauca, Plumeria alba, Prosopis juliflora, Serjania polyphylla, Tamarindus indica, and Tabebuia heterophylla, July 21, 1966 (LFM, JGT).

\section{ORDER ODONATA}

Suborder Zygoptera Superfamily Coenagrionidae

FAMILY COENAGRIONIDAE

14. Enallagma sp. near caecum (Hagen)

One specimen at light Dec. 9, 1960 (LFM).

15. Enallagma sp. near civile (Hagen)

One specimen at light Dec. 9, 1960 (LFM). 
Suborder Anisoptera

Superfamily Libelluloidea

FAMILY LIBELLULIDAE

16. Erythrodiplax umbrata (L.)

Seventeen specimens (6 males and 11 females), Acc. Nos. 242-59 and 243-59, May 27-30, 1959 and June 23, 1959 (LFM, SMG and EAJ).

17. Orthemis ferruginea $\mathrm{F}$.

Three specimens, Acc. Nos. 244-59 and 246-59 (LFM, SMG and EAJ).

18. Tramea abdominalis (Rambur)

One specimen, Acc. No. 247-59, May 27-30, 1959 (LFM and SMG).

19. Tramea onusta Hagen

One specimen, Acc. No. 245-59, June 23, 1959 (LFM and SMG).

ORDER NEUROPTERA

Suborder Planipennia

Superfamily Hemerobioidea

FAMIIY CHRYSOPIDAE

20. Chrysopa sp.

Fourteen specimens at lights, Acc. Nos. 252-59, May 27-30, 1959 and Dec. 9, 1960 (LFM and EAJ).

Superfamily Myrmeleontoidea

FAMILY MYRMELONTIDAE

21. Psammoleon bistichus (Hagen)

Twelve specimens at lights, Acc. Nos. 251-59, May 27-30, 1959 (LFM, SMG and EAJ).

\section{FAMILY ASCALAPHIDAE}

22. Ululodes opposita Banks

Nine specimens, Acc. Nos. 249-59 (4 males) and Acc. Nos. 250-59 (5 females) at lights except for one specimen, May 27-30, 1959 (LFM, SMG and EAJ). 
ORDER THYSANOPTERA

Suborder Terebrantia

FAMILY MEROTHRIPIDAE

23. Merothrips lacotae Medina Gaud

Described by Medina Gaud (11) from holotype (male) and paratype (female), Acc. No. 157-59, deposited in the Station Collection collected from ground litter composed mostly of dried leaves; extracted by Berlese funnel May 29, 1959 (SMG).

FAMILY THRIPIDAE

24. Frankliniella difficilis (Franklin)

Four specimens collected on Guilandina crista flowers in association with other thrips species, namely: $F$. insularis (Franklin), Acc. Nos. 150-59 and 155-59, May 5 and May 27, 1959, respectively (SMG).

25. Frankliniella insularis (Franklin)

Four specimens collected on Caesalpinia crista and Tabebuia heterophylla flowers, Acc. Nos. 149-59 and 155-59, May 5 and May 27, 1959 (SMG).

Suborder Tubulifera

FAMILY PHLAEOTHRIPIDAE

26. Adraneothrips alternatus Hood

Six females and 4 males extracted from ground litter May 29, 1959 (SMG).

27. Amphibolothrips (Trachythrips) wirshingi Medina Gaud

Described by Medina Gaud (11) from holotype (female) and 18 paratypes (females), allotype (male) and 8 paratypes (males), Acc. Nos. 157-59 deposited in the Station Collection, extracted from ground litter by Berlese funnel May 29, 1959 (SMG). 


\title{
ORDER HOMOPTERA
}

\author{
Suborder Auchenorrhyncha \\ Superfamily Cicadoidea
}

FAMILY CICADIDAE

\section{Proarna hilaris (Germar)}

Ten specimens collected at lights on the northcast shore of Caja de Muertos Island near the east end, Acc. Nos. 77-59, 78-59 and 79-59, May 28, 1959 (LFM and SMG); one specimen on Lantana involucrata, June 24, 1959 , and nine cast skins collected on top of sandy soil along trail on north coast.

\section{Superfamily Cicadelloidea}

FAMILY CICADELLIDAE

Subfamily Tettigellinae

29. Carneocephala sagittifera (Uhler)

Three specimens collected on weeds, Acc. No. 102-59, May 27-30, 1959 (LFM and SMG). Previously recorded by Caldwell and Martorell (4) on weeds, 1 specimen, Dec. 5, 1947.

30. Hortensia similis (Walker)

Recorded previously by Caldwell and Martorell (4) on weeds Dec. 5, 1947.

31. Poeciloscarta laticeps Metcalf \& Bruner

Previously recorded by Caldwell and Martorell (4) two specimens from weeds Dec. 5, 1947.

Subfamily Jassinae

32. Gypona puertoricensis Caldwell and Martorell

Twenty specimens collected while beating weeds and shrubs along trail on north coast from east point to lighthouse area, Acc. Nos. 82-59 and 94-59. One specimen collected at light June 24, 1959 (LFM and SMG). Previously recorded paratypes from Caja de Muertos Island by Caldwell and Martorell (4) breeding abundantly on its favorite host plant Volkameria aculeata (F.). 
33. Xerophloea viridis (F.)

Two specimens collected from weeds, Acc. No. 92-59, May 27-30, 1959 (LFM and SMG). Recorded previously by Caldwell and Martorell (4) from weeds Dec. 5, 1947.

Subfamily Agallinae

34. Agalliana sticticollis (Stål)

Recorded by Caldwell and Martorell (4) from weeds Dec. 5, 1947.

Subfamily Dorydiinae

35. Spangbergiella vulnerata convexa Lawson

Five specimens recorded by Caldwell and Martorell (4) from weeds Dec. 5, 1947.

Subfamily Euscelinae

36. Acinopterus angulatus Lawson

Recorded by Caldwell and Martorell from weeds Dec. 5, 1947.

37. Chlorotettix tethys Van Duzee

Nineteen specimens collected, 5 at lights, the remainder by beating weeds and shrubbery in the area along the north coast May 27-30, 1959 (LFM and SMG). New record for Caja de Muertos Island.

38. Chlorotettix minimus Baker

Specimen from weeds collected Dec. 11, 1947.

39. Deltocephalus flavicosta Stål

Recorded by Caldwell and Martorell (4) from weeds, grasses and shrubs Dec. 5, 1947.

40. Deltocephalus maculellus Osborn

Recorded by Caldwell and Martorell (4), from weeds Dec. 5, 1947.

41. Deltocephalus sonorus Ball

Recorded by Caldwell and Martorell (4) from weeds Dec. 5, 1947.

42. Exitianus exitiosus (Uhler)

One specimen collected from weeds, Acc. 102-59, May 27-30, 1959 (LFM and SMG). 
43. Graminella cognita Caldwell

One specimen collected by beating weeds along the north coast, Acc. No. 86-59, May 27-30, 1959 (LFM and SMG).

44. Scaphytopius alboscutellus Caldwell \& Martorell

Previously described by Caldwell and Martorell (4) from male holotype and paratypes collected Nov. 5, 1947 (LFM).

Subfamily Balcluthinae

45. Nesosteles incisa (Matsumura)

One specimen collected by beating weeds along the north coast, May 27-30, 1959, Acc. No. 86-59 (LFM and SMG). Another specimen in the Station Collection, Caldwell and Martorell, Dec. 5, 1947.

Subfamily Cicadellinae

46. Dikraneura aurulenta Lawson

Two specimens collected Dec. 11, 1947 (Caldwell and Martorell).

47. Dikraneura pseudomarginella Caldwell and Martorell

Recorded by Caldwell and Martorell (4) on "zarzilla," Leucaena glauca, Dec. 5, 1947.

48. Empoasca dilitara DeLong and Davidson

One specimen collected among weeds Dec. 5, 1947 and recorded by Caldwell and Martorell (4).

49. Empoasca fabalis DeLong

Recorded by Caldwell and Martorell (4) from weeds Dec. 11, 1947.

50. Empoasca obliqua Caldwell and Martorell

Paratypes from Caja de Muertos Island recorded by Caldwell and Martorell (4).

\section{Protalebra maculata Baker}

One female specimen recorded by Caldwell and Martorell (4), from weeds Dec. 5, 1947 (LFM).

52. Rabela tabebuiae (Dozier)

Specimens collected from weeds Dec. 5, 1947 (LFM and J. S. Caldwell) and by Martorell Dec. 11, 1947. Recorded as Protalebra tabebuiae by Caldwell and Martorell (4). 


\section{Superfamily Fulgoroidea}

\section{FAMILY ACANALONIDAE}

53. Acanalonia vivida coniceps Osborn

Recorded by Caldwell and Martorell (4).

FAMILY ACHILIDAE

54. Catonia arida Caldwell and Martorell

Recorded by Caldwell and Martorell (5) Dec. 11, 1947, paratypes from Caja de Muertos Island.

55. Catonia cinerea Osborn

Recorded by Caldwell and Martorell (5) on weeds Dec. 5, 1947, paratypes from Caja de Muertos Island.

\section{FAMILY CIXIDAE}

56. Microledrida arida Caldwell and Martorell

Originally described from male holotype and female allotype as recorded by Caldwell and Martorell (5), Dec. 11, 1947.

57. Oliarus complectus Ball

Recorded by Caldwell and Martorell (5) Dec. 11, 1947.

\section{FAMILY DELPHACIDAE}

58. Anchidelphax havanensis (Crawford)

Recorded by Caldwell and Martorell (5) as Delphacodes havanenis, from grasses Dec. 5, 1947.

59. Delphacodes nigra (Crawford)

Eight specimens collected Dec. 11, 1947 by Caldwell and Martorell (5).

60. Delphacodes propinqua (Fieber)

Recorded by Caldwell and Martorell (5).

61. Sogatodes approximatus (Crawford)

One specimen collected Dec. 5, 1947. 


\section{Cyarda salina (Dozier)}

Twelve specimens collected among weeds, Acc. Nos. 95-59 and 103-59, May 27-30, 1959 (LFM, SMG). Recorded previously by Caldwell and Martorell (5) on Batis maritima, Dec. 5, 1947.

63. Flatoidinus spinosus Caldwell

Eight specimens at lights on northeast end of the Island; 2 collected on foliage of Cocoloba uvifera, remainder among weeds, Acc. Nos. 96-59, 99-59 and 101-59, May 27-30, 1959 (LFM, SMG). New record for Caja de Muertos Island and for Puerto Rico proper.

64. Melormenis antillarum (Kirkaldy)

Recorded by Caldwell and Martorell (5) Dec. 5, 1947.

65. Melormenis dipuncta Caldwell and Martorell

Seven specimens collected among weeds, Acc. Nos. 88-59, 98-59, May 27-30, 1959. Described by Caldwell and Martorell (5) from male holotype, female allotype and paratypes collected Dec. 11, 1947 on Caja de Muertos Island.

66. Petrusa marginata (Brunnich)

One specimen collected by beating weeds, Acc. No. 104-59, May 27-30, 1959 (LFM, SMG). Previously recorded by Caldwell and Martorell (5) Dec. 5 and 11, 1947.

\section{FAMILY ISSIDAE}

67. Colpoptera maculifions Muir

Five specimens collected by beating weeds and shrubs, Acc. No. 100-59, May 27-30, 1959 (LFM, SMG).

68. Colpoptera maculifrons maculata Dozier

Previously recorded by Caldwell and Martorell (5) Dec. 5 and 11, 1947.

\section{FAMILY KINNARIDAE}

69. Quilessa wirshingi Ramos

Nine specimens collected by beating weeds, Acc. Nos. 87-59 and 91-59, May 27-30, 1959. Previously recorded by Ramos (12), described from male holotype, female allotype and paratypes collected Dec. 5, 1947 by Caldwell and L. F. Martorell on Caja de Muertos Island. 
FAMILY TROPIDUCHIDAE

70. Neurotmeta viridis (Walker)

Eighteen specimens collected by beating weeds, shrubs and trees along trail on the north coast from east point toward lighthouse area, Acc. Nos. 83-59 and 84-59, May 27-30, 1959 (LFM, SMG). Previously recorded by J. S. Caldwell and L. F. Martorell (5), Dec. 5 and 11, 1947. This species is common on the foliage of sea-grape, Coccoloba uvifera.

71. Tangella schaumi (Stål)

Eleven specimens collected by beating weeds, shrubs and trees along the northcoast trail, Acc. Nos. 83-59, 84-59 and 90-59, May 27-30, 1959 (LFM, SMG). Previously recorded by Caldwell and Martorell (5) from weeds and shrubs Dec. 5 and 11, 1947. This species seems to be common on the foliage of Coccoloba uvifera.

Suborder Stenorrhyncha

Superfamily Psylloidea

FAMILY PSYLLIDAE

72. Carsidara concolor Crawford

Previously recorded by Caldwell and Martorell (6) Dec. 11, 1947.

Superfamily Aphidoidea

FAMILY APHIDIDAE 6

73. Aphis coreopsidis Thomas

Specimens collected on tender shoots of Boerhaavea erecta and Iresine sp., Acc. No. 59-302 and 59-306, May 27-30, 1959 (LFM).

\section{Aphis gossypii Glover}

Specimens collected on tender shoots of Coccolobis diversifolia, hundreds parasitized by minute wasps, possibly Aphidius testaceipes Cresson, Acc. No. 59-300, May 27, 1959 (LFM), and on Capraria biflora (Acc. No. 60-109) April 23, 1960 (CFS, LFM, MEP, and Roy Woodbury). Aptera: all shades of green; winged: yellow abdomen with brown thorax, others dark-green body, all with wings clear.

6 The material listed under this Family is recorded in Smith, Martorell and Pérez Escolar (14). 
75. Aphis illinoiensis Shimer

On Cissus sicyoides, Acc. No. 59-310, May 29, 1959 (LFM).

76. Aphis nerii Boyer de Fonscolombe

Fairly large yellow aphid, aptera abundant on one Calotropis procera plant, Acc. No. 59-307, May 28, 1959 (LFM).

77. Aphis craccivora Koch

Aptera light green on Indigofera suffruticosa, many parasitized, Acc. No. 59-301; abundant on Capparis flexuosa, Acc. No. 59-304, May 27, 1959 (LFM). Also on Coccolobis obtusifolia, Acc. No. 60-110, April 23, 1960 (CFS, LFM, MEP). On Canavalia maritima, Acc. No. 60-112, Apr. 23, 1960 (CFS, LFM, MEP and Roy Woodbury).

78. Aphis spiraecola Patch

Green aptera on tender shoots of Boerhaavea erecta, Acc. No. 59-302; in association with $A$. coreopsidis, Acc. 59-303; abundant on tender shoots of Bursera simaruba, Acc. No. 59-305. Winged forms abundant, curling the tender shoots of Rauwolfia lamarckii, May 27, 1959 (LFM); abundant on Exostema caribaeum, Acc. No. 59-309, May 28, 1959 (LFM); abundant on Croton humilis and Erithalis fruticosa, May 29, 1959 (LFM).

Superfamily Coccoidea

FAMILY COCCIDAE

79. Saissetia nigra (Nietner)

On branches of the common weed Malvastrum spicatum May 28, 1959 (LFM, SMG).

80. Saissetia oleae (Bernard)

On branches and trunk of Malvastrum spicatum May 28, 1959 (LFM, SMG).

\section{FAMILY DIASPIDIDAE}

81. Pinnaspis strachani (Cooley)

On branches and trunk of Malvastrum spicalum May 28, 1959 (LFM, SMG). 
FAMILY MARGARODIDAE

82. Margarodes formicarium Guilding

Ground pearls in soil under stones in trail near animal yard May 28, 1959 (LFM).

83. Icera purchasii Maskell

On Chamaecrista granulata, collected by the late Juan Alberto Wirshing on Cajanus cajan. Also observed on Citrus limonia May 28, 1959 (SMG).

FAMILY ORTHEZIIDAE

84. Orthezia insignis Browne

From branches of Coccoloba diversifolia and Stigmaphyllon sp. May 28, 1959, (LFM, SMG).

FAMILY PSEUDOCOCCIDAE

85. Ferrisia virgata (Cockerell)

Specimens observed Dec. 9, 1960 (LFM).

ORDER HEMIPTERA

Suborder Gymnocerata

Superfamily Cimicoidea

FAMILY MIRIDAE

86. Phytocoris brunneus Maldonado

Described as new by Maldonado-Capriles (10) from 13 specimens collected at light May 1959 (LFM, SMG, EAJ).

Superfamily Reduvioidea

FAMILY REDUVIIDAE

87. Pnirotis infivma Stål

One specimen collected Dec. 11, 1947, Acc. No. 154-47.

Superfamily Lygaeoidea

FAMILY LYGAEIDAE

88. Nysius ericae (Schilling)

Nine specimens, Acc. No. 134-47, 136-47 and 137-47, Dec. 11, 1947. 
Superfamily Coreoidea

FAMILY COREIDAE

89. Catorhintha guttula (F.)

One specimen collected Nov. 1947, Acc. No. 273-47 (LFM).

90. Leptoglossus concolor (Walker)

One specimen collected, Acc. No. 156-59, May 24, 1959 (LFM, SMG, EAJ).

\section{FAMILY RHOPALIDAE}

91. Liorhyssus hyalinus (F.)

Three specimens, Acc. No. 271-47, Nov. 1947 (LFM).

FAMILY ALYDIDAE

92. Megalotomus rufipes (Westwood)

Six specimens collected at lights May 27-30, 1959 (LFM, SMG) and Dec. 9, 1960 (LFM).

\section{Superfamily Pentatomoidea}

FAMILY PENTATOMIDAE

93. Acrosternum marginatum (Palisot de Beauvois)

One specimen collected Acc. No. 290-47, Nov. 1947 (LFM); three specimens collected at lights May 29, 1959 and June 24, 1959 (LFM, SMG).

94. Diolcus disjunctus Barber

Two specimens collected, Acc. No. 266-47, Nov. 1947 (LFM); sixteen specimens collected May 24, 27-30, 1959 (LFM, SMG, EAJ).

95. Mormidea cubrosa Dallas

Two specimens, Acc. Nos. 155-59 and 157-59, May 24, 1959 (LFM, SMG, EAJ).

96. Nezara viridula (L.)

Two specimens collected May 29, 1959 and June 24, 1959, one at lights (SMG, LFM).

97. Piezodorus guildinii (Westwood)

Three specimens, Acc. Nos. 267-47 and 288-47, collected Nov. 1947 (LFM); one specimen collected at light Dec. 9, 1960 (LFM). 
98. Thyanta antiguensis (Westwood)

Nine specimens, Acc. No. 269-47, Nov. 1947 (LFM).

99. Thyanta casta Stål

Recorded by Wolcott (16) as collected by Prof. J. A. Ramos.

100. Thyantha perditor (F.)

Four specimens, Acc. No. 154-59, collected May 27-30, 1959 and Dec. 9, 1960 (LFM, EAJ).

\section{ORDER COLEOPTERA}

Suborder Polyphaga

Superfamily Staphylinoidea

FAMILY STAPHYLINIDAE

101. Xantholinus sp.

One specimen, Acc. No. 157-47, Dec. 11, 1947, in the Station Collection. Superfamily Scarabaeoidea

FAMILY SCARABAEIDAE

102. Phyllophaga n. sp.

Twenty-one specimens, Acc. No. 126-59, collected May 27-30, 1959, 4 on Tabebuia heterophylla, 4 on Sarcomphallus reticulatus and 13 at light (LFM, SMG, EAJ). Nine specimens sent to Dr. M. Sanderson at the Illinois Natural History Survey, Urbana, Ill. U.S.A. for identification and he determined this species as undescribed.

103. Phyllophaga spp.

Fifty-one specimens, Acc. No. 125-59, collected May 27-30, 1959; 9 on various known hosts, 11 on Coccolobis diversifolia, 13 on Coccolobis uvifera, 9 on Ficus laevigata and 9 at light (LFM, SMG, EAJ). Twenty seven specimens were sent to Dr. M. Sanderson as indicated above.

Superfamily Buprestoidea

FAMILY BUPRESTIDAE

104. Acmaeodera gundlachi Fisher

Five specimens, Acc. No. 110-59, three collected May 27-30, 1959 and two June 24, 1959 (LFM, SMG, EAJ and AAA). 
105. Chrysobothris megacephala Laporte \& Gory

One specimen, Acc. No. 109a-59, May 27-30, 1959 (LFM, SMG).

106. Chrysobothris thoracica (F.)

Three specimens, Acc. No. 111a-59, May 27-30, 1959 (LFM, SMG, EAJ).

\section{Superfamily Elateroidea}

FAMILY ELATERIDAE

107. Conoderus sp. prob. bifoveatus (Palisot)

Fifty specimens at lights May 5, 24 and 28, 1959 and June 24, 1959 (LFM, SMG).

108. Aeolus elegans (F.)

One specimen at light Dec. 9, 1960 (LFM).

\section{Superfamily Cantharoidea}

FAMILY LAMPYRIDAE

109. Photinus vittatus Olivier

One specimen at light May 27-30, 1959 (LFM, SMG).

\section{Superfamily Cucujoidea}

FAMILY COCCINELLIDAE

\section{Coleophora inaequalis (F.)}

Three specimens, Acc. Nos. 268-47 and 277-47, Nov. 1947 (LFM); three specimens, Acc. No. 117-59, May 27-30, 1959 (LFM, SMG, EAJ).

\section{Cycloneda sanguinea (L.)}

Seventeen specimens May 27-30, 1959, two May 5, 1959, and eight May 24, 1959 (LFM, SMG, EAJ).

112. Scymnus roseicollis Mulsant

Four specimens, Acc. No. 122-59, June 24, 1959 (SMG).

\section{FAMILY TENEBRIONIDAE}

113. Blapstinus sp.

Two specimens at light, Acc. No. 142-59, May 27-30, 1959 (LFM, SMG, EAJ). 


\section{Phaleria angustata Chevr.}

Five specimens, Acc. No. 134-47, Dec. 11, 1947, in the Station Collection; fifteen specimens at light, Acc. No. 145-59, May 27-30, 1959; June 24, 1959 (LFM, SMG, EAJ) and Dec. 9, 1960 (LFM, EAJ).

\section{FAMILY ALLECULIDAE}

\section{Hymenorus sp.}

Twenty five specimens, 12 at lights May 27-30, June 25, 1959 (LFM, SMG, EAJ), and Dec. 19, 1960 (LFM, EAJ), Acc. No. 144-59.

\section{FAMILY OEDEMERIDAE}

\section{Oxacis vittata (F.)}

One specimen Oct. 11, 1947, Acc. No. 133-47; fifteen specimens at light, Acc. No. 127-59, May 27-30, 1959 (LFM, SMG).

Superfamily Chrysomeloidea

FAMILY CERAMBYCIDAE

117. Eburia quadrimaculata (L.)

Ten specimens at light, Acc. No. 113-59, May 27-30, 1959 (LFM, SMG, EAJ), one specimen Dec. 9, 1960 (LFM, EAJ).

118. Elaphidion irroratum (L.)

One specimen at light, Acc. No. 112a-59, May 27-30, 1959 (LFM, SMG, EAJ).

\section{Elaphidion mutatum Gahan}

One specimen at light Dec. 9, 1960 (LFM).

120. Methia necydalea (F.)

Eight specimens at light May 27-30, 1959 (LFM, SMG, EAJ).

FAMILY CHRYSOMELIDAE

\section{Chaetocnema sp.}

Three specimens, Acc. No. 154-47, Dec. 11, 1947.

122. Cryptocephalus perspicax Weise

Three specimens, Acc. No. 138-47, Dec. 11, 1947, in Station Collection; five specimens at light, Acc. No. 118-59, May 27-30, 1959 and June 24, 1959 (LFM, SMG, EAJ). 
123. Cryptocephalus sp.

One specimen, Acc. No. 119-59, May 27-30, 1959 (LFM, SMG, EAJ).

124. Hilarocassis exclamationis $\mathrm{L}$.

Forty six specimens, Acc. No. 124-59, June 24, 1959 (LFM, SMG, EAJ).

125. Metriona sp.

One specimen, Acc. No. 121-59, May 27-30, 1959 (SMG).

126. Podagrica cyanipennis Weise

Three specimens at light, Acc. No. 123-59, May 27-30, 1959 (LFM, SMG, EAJ).

\section{Superfamily Curculionoidea}

FAMILY CURCULIONIDAE

127. Anchonus suillus (F.)

One specimen, Acc. No. 129-47, on Dec. 11, 1947 in Station Collection. 128. Apodrosus sp. near argentatus Wolcott

Three specimens in the Station Collection, Acc. No. 132-47; five specimens, Acc. No. 132-59, May 27-30, 1959 (LFM, SMG, EAJ).

129. Diaprepes abbreviatus (L.)

Three specimens, Acc. No. 130-59, May 27-30, 1959 (LFM, SMG, EAJ). 130. Lachnopus coffeae Marshall

One specimen, Acc. No. 142-47, Dec. 11, 1947, in Station Collection.

\section{ORDER DIPTERA}

Superfamily Culicoidea

FAMILY CULICIDAE

131. Aedes (Ochlerotatus) sollicitans (Walker)

Two larvae, apparently this species, collected in water in a hole in a rock Dec. 9, 1969 (LFM, EAJ). 


\section{Superfamily Tabanoidea}

FAMILY TABANIDAE

132. Stenotabanus (Aegialomyia) nervosus Curran

One specimen, Acc. No. 256-47, Nov. 1947 (LFM); two specimens, Acc. No. 177-59, May 27-30, 1959 (LFM, SMG, EAJ).

133. Stenotabanus (Aegialomyia) stigma (F.)

Five specimens, Acc. No. 161-59, May 27-30, 1959 (LFM, SMG, EAJ). Superfamily Asiloidea

FAMILY ASILIDAE

134. Ommatius marginellus (F.)

One specimen, Acc. No. 161-59, at light May 27-30, 1959 (LFM, SMG, EAJ).

135. Anthrax sp.

Three specimens, Acc. No. 164-59 and 165-59, two at light May 27-30, 1959 (LFM, SMG, EAJ).

136. Villa gorgon (F.)

One specimen at light May 28, 1959 (LFM, SMG, EAJ).

Superfamily Syrphoidea

FAMILY SYRPHIDAE (15)

137. Allograpta limbata (F.)

One specimen at light, Acc. No. 166-59, May 27-30, 1959 (LFM, SMG, EAJ).

138. Baccha clavata (F.)

One specimen, Acc. No. 275-47, Nov. 1949 in Station Collection.

139. Baccha (Ocyptamus) cylindrica (F.)

Three specimens at light, Acc. No. 163-59, May 27-30, 1959 (LFM, SMG, EAJ).

140. Volucella unipunctata Curran

Specimens collected Nov. 1947 (LFM), Acc. No. 272-47; May 27-30, 1959, Acc. No. 173-59 (LFM, SMG, EAJ). 


\section{Superfamily Muscoidea}

\section{FAMILY MUSCIDAE}

141. Musca domestica (L.)

One male specimen at light, Acc. No. 175-59, May 27-30, 1959 (LFM, SMG).

\section{ORDER LEPIDOPTERA}

\section{Superfamily Tineoidea}

FAMILY TINEIDAE

142. Tineola sp.

Bags of larvae observed on walls at lighthouse building May 27-30, 1959 (LFM).

\section{Superfamily Gelechoidea}

FAMILY ETHMIIDAE

143. Ethmia confusella (Walker)

Two specimens at light, Acc. No. 184-59, May 28, 1959 (LFM, SMG).

144. Ethmia notatella (Walker)

Five specimens at light, Acc. No. 185-59, May 28, 1959 (LFM, SMG).

Superfamily Tortricoidea

FAMILY OLETHREUTIDAE

145. Laspeyresia flavicollis (Walshingham)

Four specimens at light, Acc. No. 195-59, May 28, 1959 (LFM, SMG, EAJ).

FAMILY COSSIDAT

146. Psychonoctua personalis Grote

One specimen at light, Acc. No. 182-59, May 29, 1959 (LFM, SMG). The larvae bore into the twigs of numerous species of trees, two of which are found on Caja de Muertos Island, namely: sea grape, Coccoloba uvifera and "mangle bobo," Laguncularia racemosa. 
Superfamily Pyraloidea

FAMILY PYRAUSTIDAE

147. Dichogama redtenbacheri Ledercr

One specimen, Acc. No. 186-59, May 25, 1959 (LFM, SMG, EAJ).

148. Hymenia perspectalis (Hübner)

One specimen at light, Acc. No. 216-59, May 27-30, 1959 (LFM, SMG, EAJ).

149. Margaronia costata (F.)

Twenty-two specimens at light, Acc. No. 188-59, May 27-30, 1959 (LFM, SMG).

150. Maruca testulalis (Geyer)

One specimen at light, Acc. No. 191-59, May 27-30, 1959, (LFM, SMG, EAJ).

151. Pilocrocis inguinalis (Guenée)

Six specimens at lights, Acc. No. 197-59, 212-59 and 229-59, May 27-30 1959 (LFM, SMG, EAJ).

152. Pilocrocis tripunctata (F.)

Five specimens, Acc. No. 187-59, May 27-30, 1959 (LFM, SMG, EAJ). 153. Synclera traducalis (Zeller)

One specimen, Acc. No. 213-59, at light, May 27-30, 1959 (SMG, LFM, EAJ).

154. Zinckenia fascialis (Cramer)

Two specimens May 27-30, 1959 (LFM, SMG).

FAMILY CHRYSAUGIDAE

155. Pachymorphus subductellus Möschler

Two specimens, Acc. No. 190-59, at light, May 27-30, 1959 (LFM, SMG, EAJ).

\section{FAMILY PHYCITIDAE}

156. Cactoblastis cactorum (Berg.)

Recorded by García Tudurí et al. (7) during their termite survey studies in that locality in 1966 as attacking the host plants Opuntia dillenii and Consolea rubescens. 
Superfamily Geometroidea

FAMILY GEOMETRIDAE

157. Iridopsis momaria (Guenée)

One specimen at light, Acc. No. 190-59, May 27-30, 1959 (LFM, SMG, EAJ).

158. Macaria diffusata Guenée

Five specimens at light May 27-30, 1959 (LFM, SMG, EAJ).

159. Pterocypha defensata Walker

Two specimens, Acc. No. 219-59, May 27-30, 1959 (LFM, SMG, EAJ).

160. Semaeopus caecaria (Hübner)

Eight specimens at light, Acc. No. 199-59, May 27-30, 1959 (LFM, SMG).

\section{Superfamily Noctuoidea}

FAMILY CTENUCHIDAE

161. Horama panthalon (F.)

Eight specimens at light, Acc. No. 181-59, May 28, 1959 (LFM, SMG).

162. Horama pretus (Cramer)

Two specimens at light, Acc. No. 180-59; larvae observed feeding on foliage of "coscorrón," Elaeodendron xylocarpum, a common shrub on the Island, May 28, 1959 (LEM, SMG and EAJ).

FAMILY ARCTIIDAE

163. Utethesia ornatrix stretchii (Butler)

One specimen, Acc. No. 179-59, collected among weeds May 28, 1959 (SMG).

\section{FAMILY NOCTUIDAE}

164. Casandria abseuzalis (Walker)

One specimen at light, Acc. No. 192-59, May 27-30, 1959 (LFM, SMG, EAJ). 
165. Catabena vitrina (Walker)

Eleven specimens at light, Acc. No. 182-59, May 27-30, 1959 (LFM, SMG).

166. Feltia subterranea (F.)

Two specimens at light, Acc. No. 223-59 and 226-59, May 27-30, 1959 (LFM, SMG, EAJ).

167. Heliothis virescens (F.)

Two specimens at light, Acc. No. 194-59, May 27-30, 1959 (LFM, SMG).

168. Melipolis famelica (Guenée)

Fourteen specimens at light, Acc. No. 237-59, May 27-30, 1959 (LFM, SMG, EAJ).

169. Melipotis fasciolaris (Hübner)

Four specimens at light, Acc. No. 235-59 and 236-59, May 27-30, 1959 (LFM, SMG, EAJ).

170. Meridyrias progenies (Guenéc)

Two specimens at light, Acc. No. 193-59, May 27-30, 1959 (SMG, LFM, EAJ).

171. Noropsis hieroglyphica (Cramer)

Ten specimens at light, Acc. No. 189-59, May 27-30, 1959 (LFM, SMG, EAJ).

172. Spodoptera eridania (Cramer)

One specimen at light, Acc. No. 225-59, May 27-30, 1959 (LFM, SMG, EAJ).

173. Spodoptera frugiperda (J. E. Smith)

Two specimens at light, Acc. No. 224-59 and 227-59, May 27-30, 1959 (LFM, SMG, EAJ).

174. Xylomiges sunia Guenée

One specimen at light May 27-30, 1959 (LFM, SMG).

FAMILY PERICOPIDAE

175. Composia sybaris (Cramer)

One specimen at light, Acc. No. 178-59, May 29, 1959 (LFM, SMG). 
Superfamily Sphingoidea

FAMILY SPHINGIDAE

176. Aellopos tantalus zonata (Drury)

One specimen, Acc. No. 183-59, May 27-30, 1959 (LFM, SMG).

177. Cautethia noctuiformis (Walker)

Two specimens at light, Acc. No. 196-59, May 27-30, 1959 (LFM, SMG).

178. Celerio lineata lineata (F.)

Three specimens at light, Acc. No. 238-59, May 27-30, 1959 (LFM, SMG, EAJ).

179. Pseudosphinx tetrio (L.)

Many caterpillars observed attacking foliage of Plumiera alba on May 27-30, 1959 (LFM, SMG).

Superfamily Hesperoidea

FAMILY HESPERIIDAE

180. Ephyriades arcas (Drury)

Nine specimens (5 males and 4 females), Acc. No. 239-59, May 27-30, 1959. (LFM, SMG, EAJ).

181. Pyrgus syrichtus (F.)

One specimen May 27-30, 1959 (LFM, SMG, EAJ).

182. Urbanus dorantes cramptoni Comstock

One specimen May 27-30, 1959 (LFM, SMG).

Superfamily Papilionoidea

FAMILY PIERIDAE

183. Appias (Glutophrissa) drusilla boydi Comstock

One specimen May 27-30, 1959 (LFM, SMG).

184. Appias (Glutophrissa) drusilla (Cramer)

Three specimens May 27-30, 1959 (LFM, SMG).

185. Ascia monuste eubotea (Latreille)

Eight specimens May 27-30, 1959 (LFM, SMG). 
186. Ascia monuste monuste (L.)

Twelve specimens May 27-30, 1959 (LFM, SMG).

187. Ascia monuste phileta (F.)

Three specimens May 27-30, 1959 (LFM, SMG).

188. Ascia monuste virginia (Latreille)

Seventeen specimens May 27-30, 1959 (LFM, SMG).

189. Phoebis (Phoebis) sennae sennae (L.)

Three specimens May 27-30, 1959 (LFM, SMG).

190. Kricogonia lyside (Latreille)

One specimen May 27-30, 1959, (LFM, SMG).

FAMILY NYMPHALIDAE

191. Heliconius charitonius charitonius (L.)

One specimen, Acc. No. 241-59, May 27-30, 1959 (LFM, SMG).

192. Junonia evarete zonalis (C. and R. Felder)

Two specimens May 27-30, 1959 (LFM, SMG).

FAMILY LYCAENIDAE

193. Hemiargus (Cyclargus) noeli (Comstock \& Huntington)

Six specimens, Acc. No. 240-59, May 27-30, 1959 (LFM, SMG).

194. Hemiargus (Hemiargus) hanno watsoni Comstock \& Huntington On May 27-30, 1959 (LFM, SMG).

ORDER HYMENOPTERA

Suborder Apocrita

Superfamily Ichneumonoidea

FAMILY BRACONIDAE

195. Rogas sp.

One specimen, Acc. No. 120-47, Dec. 11, 1947 in Station Collection. 


\section{FAMILY ICHNEUMONIDAE}

196. Enicospilus concolor (Cresson)

Two specimens at light Dec. 9, 1960 (LFM).

\section{Superfamily Chalcidoidea}

FAMILY CHALCIDIADE

197. Brachymeria incerta (Cresson)

One specimen, Acc. No. 290-59, May 24, 1959 (LFM, SMG, EAJ).

Superfamily Vespoidea

FAMILY VESPIDAE

198. Mischocyttarus cubensis (Saussure)

Nine specimens, Acc. No. 281-59, May 27-30, 1959 (LFM, SMG, EAJ). 199. Pachodynerus atratus (F.)

Two specimens, Acc. No. 239-59, May 27-30, 1959 (LFM, SMG, EAJ) 200. Polistes major Palisot de Beauvois

Ten specimens, Acc. No. 279-59, May 27-30, 1959 (LFM, SMG, EAJ). 201. Polistes americanus F.

Three specimens, Acc. No. 280-59, May 27-30, 1959 (LFM, SMG, EAJ) FAMILY POMPILIDAE

202. Pepsis formosa Say

Eleven specimens, Acc. No. 282-59, May 27-30, 1959 (LFM, SMG, EAJ). Recorded previously by Wolcott (16) as collected by Prof. J. A. Ramos.

Superfamily Sphecoidea

FAMILY SPHECIDAE

203. Sceliphron caementarium (Drury)

Two specimens, Acc. No. 278-59, May 27-30, 1959 (LFM, SMG, EAJ).

Superfamily Apoidea

FAMILY MEGACHILIDAE

204. Megachile (Archimegachile) lanata (F.)

One specimen, Acc. No. 277-59, May 27-30, 1959 (LFM, SMG). 
205. Bembix ciliata F.

Four specimens June 24, 1959 (LFM, SMG).

206. Bicyrtes spinosd (F.)

Two specimens June 24, 1959 (LFM, SMG).

207. Centris versicolor (F.)

Twelve specimens, Acc. No. 276-59, hovering about steep slope, May 27-30, 1959.

208. Microbembex monodonta (Say)

Six specimens May 27-30, 1959 (LFM, SMG, EAJ).

209. Stictia signata (L.)

One specimen, Acc. No. 294-59, May 27-30, 1959 (LFM, SMG, EAJ). It is a very common local species.

210. Xylocopa brasilianorum (L.)

Five specimens, Acc. No. 275-59, May 27-30, 1959 (LFM, SMG, EAJ).

\section{SUMMARY}

This report is an annotated checklist of 210 species of insects, representing 11 orders and 79 families, which have been recorded for Caja de Muertos, a small island located off the coast of Puerto Rico about 8 miles southeast of Ponce harbor. A brief account is given of the various, mainly entomological, scientific explorations conducted thereon.

\section{RESUMEN}

Este trabajo consiste de una lista catalogada y anotada de 210 especies de insectos, representando 11 órdenes y 79 familias, que han sido informadas como existentes en Caja de Muertos, pequeña isla localizada a 8 millas del sudeste de la bahía de Ponce (en la costa sur de Puerto Rico). Contiene también un breve resumen de las diversas exploraciones científicas (mayormente entomológicas) que se han realizado en esa isla.

\section{LITERATURE CITED}

1. Britton, N. L., Further botanical exploration in Porto Rico, J.N.Y. Bot. Gard. 16: 103-12, 1915.

2. -, History of the survey, Sci. Surv. P.R. and the V. Ids. N.Y. Acad. Sc. 1(1): 1-10, 1919.

3. - , and Wilson, P., Botany of Porto Rico and the Virgin Islands-Descriptive Flora-Spermatophyta, Sci. Surv. Porto Rico \& Virgin Ids., 5: pp. 663, 1923-1930. 
4. Caldwell, J. S., and Martorell, L. F., Review of the Auchenorynchous Homoptera of Puerto Rico, Part I. Cicadellidae, J. Agr. Univ. P.R. 34(1): 1-132, 1950.

5. - - - Review of the Auchenorynchous Homoptera of Puerto Rico, Part II. The Fulgoroidea except Kinnaridae, J. Agr. Univ. P.R. 34(2) : 133-269, 1950.

6. -, -, A brief review of the Psylliidae of Puerto Rico (Homoptera), Ann. Ent. Soc. Amer. 44(4): 603-13, 1951.

7. Garcia Tuduri, J. C., Martorell, L. F., and Medina Gaud, S., Geographical distribution and host plants of the cactus moth, Cacloblastis cactorum (Berg) in Puerto Rico and the United States Virgin Islands, J. Agr. Univ. P.R. 55(1): $130-4,1971$.

8. Grant, C., and Roosevelt, C., The herpetology of Caja de Muertos Island and Cardona Key, Porto Rico, J. Dep. Agr., 16(1): 47-9, 1932.

9. Labat, J. B., Voyage auxlles de l'Amerique, contenant l'Histoire Naturelle de ces Pays, I 'Origine, le Moeurs, la Religion et le Gouvernment des Habitants Anciens et Modernes (New Edition, 8 vols., Paris 1842) (Originally published in Paris, France, in 1724) 7: 311-14, 1842.

10. Maldonado Capriles, J., The Miridae of Puerto Rico (Insecta, Hemiptera), Agr. Exp. Sta., Univ. P.R. Tech. Paper 45, pp. 1-133, 1969.

11. Medina Gaud, S., The Thysanoptera of Puerto Rico, Agr. Exp. Sta., Univ. P.R. Tech. Paper 32, pp. 1-157, 1961.

12. Ramos, J. A., A review of the Auchenorynchous Homoptera of Puerto Rico, J. Agr. Univ. P.R. 41(1): 38-117, 1957.

13. Schmidt, K. P., Amphibians and land reptiles of Porto Rico, with a list of those reported from the Virgin Islands, Sci. Surv. P.R. and the V. Is. N.Y. Acad. Sci. V 10(1): 1-60, 1928.

14. Smith, C. F., Martorell, L. F., and Pérez-Escolar, M. E., Aphididae of Puerto Rico, Agr. Exp. Sta. Univ. P.R. Tech. Paper 37, pp. 1-121, 1963.

15. Telford, H. S., The Syrphidae of Puerto Rico, J. Agr. Univ. P.R. $57(3)$ : 217-46, 1973.

16. Wolcott, G. N., The insects of Puerto Rico, J. Agr. Univ. P.R. 32(1-4): 1-975, 1948. 